\title{
Evidence for GABA-Induced Systemic GABA Accumulation in Arabidopsis upon Wounding
}

\author{
Sandra S. Scholz ${ }^{1 t}$, Jaiana Malabarba ${ }^{2,3 t}$, Michael Reichelt ${ }^{4}$, Monika Heyer ${ }^{2}$, \\ Frank Ludewig ${ }^{5}$ and Axel Mithöfer ${ }^{2 *}$ \\ ${ }^{1}$ Institute of General Botany and Plant Physiology, Friedrich Schiller University, Jena, Germany, ${ }^{2}$ Department of Bioorganic \\ Chemistry, Max Planck Institute for Chemical Ecology, Jena, Germany, ${ }^{3}$ Graduate Program in Cell and Molecular Biology, \\ Biotechnology Center, Federal University of Rio Grande do Sul, Porto Alegre, Brazil, ${ }^{4}$ Department of Biochemistry, Max \\ Planck Institute for Chemical Ecology, Jena, Germany, ${ }^{5}$ Division of Biochemistry, Department of Biology, University of \\ Erlangen-Nuremberg, Erlangen, Germany
}

OPEN ACCESS

Edited by:

Wim Van den Ende, KU Leuven, Belgium

Reviewed by:

Barry J. Shelp,

University of Guelph, Canada

Clay Carter,

University of Minnesota, USA

*Correspondence:

Axel Mithöfer

amithoefer@ice.mpg.de

${ }^{t}$ These authors have contributed equally to this work.

Specialty section: This article was submitted to Plant Physiology, a section of the journal Frontiers in Plant Science

Received: 19 January 2017 Accepted: 07 March 2017

Published: 22 March 2017

Citation:

Scholz SS, Malabarba J, Reichelt M, Heyer M, Ludewig F and Mithöfer A

(2017) Evidence for GABA-Induced Systemic GABA Accumulation in Arabidopsis upon Wounding. Front. Plant Sci. 8:388 doi: 10.3389/fpls.2017.00388
The non-proteinogenic amino acid $\gamma$-aminobutyric acid (GABA) is present in all plant species analyzed so far. Its synthesis is stimulated by either acidic conditions occurring after tissue disruption or higher cytosolic calcium level. In mammals, GABA acts as inhibitory neurotransmitter but its function in plants is still not well understood. Besides its involvement in abiotic stress resistance, GABA has a role in the jasmonateindependent defense against invertebrate pests. While the biochemical basis for GABA accumulation in wounded leaves is obvious, the underlying mechanisms for woundinginduced GABA accumulation in systemic leaves remained unclear. Here, the Arabidopsis thaliana knock-out mutant lines pop2-5, unable to degrade GABA, and tpc1-2, lacking a wounding-induced systemic cytosolic calcium elevation, were employed for a comprehensive investigation of systemic GABA accumulation. A wounding-induced systemic GABA accumulation was detected in tpc1-2 plants demonstrating that an increased calcium level was not involved. Similarly, after both mechanical wounding and Spodoptera littoralis feeding, GABA accumulation in pop2-5 plants was significantly higher in local and systemic leaves, compared to wild-type plants. Consequently, larvae feeding on these GABA-enriched mutant plants grew significantly less. Upon exogenous application of a $\mathrm{D}_{2}$-labeled GABA to wounded leaves of pop2-5 plants, its uptake but no translocation to unwounded leaves was detected. In contrast, an accumulation of endogenous GABA was observed in vascular connected systemic leaves. These results suggest that the systemic accumulation of GABA upon wounding does not depend on the translocation of GABA or on an increase in cytosolic calcium.

Keywords: $\gamma$-aminobutyric acid, herbivory, Spodoptera littoralis, MecWorm, calcium, plant defense, systemic signaling

\section{INTRODUCTION}

The four carbon non-proteinogenic amino acid $\gamma$-aminobutyric acid (GABA) is present in a multitude of organisms and was also detected in all plant species analyzed so far (Shelp et al., 2009). In contrast to mammals - where GABA acts as inhibitory neurotransmitter by regulating ion channels - its function in plants is still not completely understood. It seems that the role of GABA 
in plants is quite diverse. Several studies indicate that GABA is not only involved in regulating metabolic pathways like the Krebs cycle, it additionally acts as a signaling molecule in plant growth and development (Bouche and Fromm, 2004; Fait et al., 2008). For example, it was reported that a tightly regulated GABA level is required for optimal root and pollen tube growth (Palanivelu et al., 2003; Mirabella et al., 2008; Renault et al., 2011). Additionally, it was shown that various abiotic as well as biotic stress stimuli induce an elevation of the GABA level in plant tissue (Bown and Shelp, 1997; Kinnersley and Turano, 2000). For example, it was observed that salt and cold stress or tissue damage of soybean (Glycine max) leaves leads to rapid accumulation of GABA up to 25-fold (Wallace et al., 1984; Ramputh and Bown, 1996). Arabidopsis thaliana plants producing a lower constitutive level of GABA showed a higher susceptibility to drought stress due to a stomata closure defect which could be rescued by increasing the internal GABA level (Mekonnen et al., 2016). Also, external application of GABA to Oryza sativa seedlings and Piper nigrum plants could enhance the individuals' performance under heat and drought stress conditions, respectively (Nayyar et al., 2014; Vijayakumari and Puthur, 2016).

Previous studies suggest that GABA acts as a player in plant defense against insect herbivores, although the exact impact of an elevated GABA concentration on the invertebrates is not clear (Ramputh and Bown, 1996; Bown et al., 2006; Scholz et al., 2015; Bown and Shelp, 2016). On the one hand, insects feeding on high concentration of GABA show a reduced performance. Rearing lepidopteran Choristoneura rosaceana cv Harris larvae on artificial diet supplemented with 1.6-2.6 $\mu \mathrm{mol}$ GABA ( $\mathrm{g}$ fresh weight $)^{-1}$ reduced growth and survival of the insects and delayed their development (Ramputh and Bown, 1996). Similar, Spodoptera littoralis larvae fed with a GABA concentration up to $1 \mu \mathrm{mol}(\mathrm{g} \text { food) })^{-1}$ gained less weight compared with the control group (Scholz et al., 2015). On the other hand, attacked plants had an elevated level of GABA in plant tissue in response to herbivore contact. The movement of crawling insects on the leaf surfaces of Nicotiana tabacum and Glycine max plants was sufficient to stimulate the local GABA level by five-fold after 5-10 min (Bown et al., 2002). In Arabidopsis thaliana Col-0 plants, feeding of $S$. littoralis larvae for $3 \mathrm{~h}$ increased the local GABA concentration by two-fold (Scholz et al., 2015). Interestingly, continuous mechanical wounding with the robotic caterpillar MecWorm (Mithöfer et al., 2005) resulted in an up to 10-fold elevation of the local endogenous GABA level (Scholz et al., 2015).

In Arabidopsis, GABA is mainly produced from L-glutamate; the decarboxylation reaction is catalyzed by glutamate decarboxylases (GADs), which are encoded by five genes. GAD1 and GAD2 are the most abundantly expressed members of this family (Shelp et al., 2012; Scholz et al., 2015). It was shown that GAD activity is induced by two different mechanisms: (i) in intact plant tissue and neutral pH GAD activity is stimulated in a $\mathrm{Ca}^{2+}$-dependent manner by binding of calmodulin to the CAM-binding site (Snedden et al., 1995); (ii) after wounding of plant cells the vacuolar content is released and the cytosol is acidified which leads to a $\mathrm{Ca}^{2+}$-independent activation of GADs (Carroll et al., 1994). The degradation of GABA is carried out in the mitochondrial matrix by a GABA-transaminase (GABA-T/POP2). In Arabidopsis, GABA-T is encoded by only one gene whose knock-out results in constitutive elevated level of GABA in the pop2-5 plant (Palanivelu et al., 2003).

In recent studies, the focus in plant wounding-induced stress signaling shifted from only analyzing the locally treated leaf to considering the whole plant response including the systemic leaves (Farmer et al., 2013; Mousavi et al., 2013; Kiep et al., 2015). When the plant was wounded or attacked by a herbivore on one leaf, a spike in cytosolic calcium, $\left[\mathrm{Ca}^{2+}\right]_{\text {cyt }}$, was generated locally as well as in adjacent leaves with vascular connections to the treated leaf. In Arabidopsis thaliana plants, wounding leaf 8 including its midrib, caused a $\left[\mathrm{Ca}^{2+}\right]_{\text {cyt }}$ elevation in vascular connected leaves 5, 8, and 13 (Dengler, 2006; Kiep et al., 2015). The response in the local leaf was immediate whereas the response in the systemic leaves showed a delay of 1-2 min. A similar response pattern was observed for plants fed by S. littoralis larvae (Kiep et al., 2015). Strikingly, the $\left[\mathrm{Ca}^{2+}\right]_{\text {cyt }}$ elevation in adjacent leaves after wounding is dependent on the presence of the $\mathrm{Ca}^{2+}$-permeable vacuolar channel TWO PORE CHANNEL 1 (TPC1-2) (Kiep et al., 2015). The systemic signaling is quite complex and the elevation of $\left[\mathrm{Ca}^{2+}\right]_{\text {cyt }}$ was accompanied by e.g., an increase in ROS and electrical signals moving to systemic tissues of the plant (Gilroy et al., 2016). Additionally, it was observed that a precursor of the defense-related phytohormone jasmonic acid (JA) was able to travel from the wounded local tissue to unwounded systemic leaves when applied exogenously (Jimenez-Aleman et al., 2015). Similarly, a systemic accumulation of GABA in unwounded adjacent leaves was detected after mechanical wounding of one single defined leaf in Arabidopsis thaliana (Scholz et al., 2015).

Thus, the open question is how insect feeding or wounding initiates the systemic accumulation of GABA in the plant. Two different mechanisms are conceivable. First, the observed systemic cytosolic calcium elevation could trigger the production of GABA in the systemic leaves by interaction of GADs with calmodulin. Second, no signaling is involved but GABA itself can travel from the treated leaf through the vascular system to the systemic leaves. Here, we addressed and challenged these hypotheses. Therefore, we used an Arabidopsis tpc1-2 mutant lacking a systemic cytosolic calcium signal after wounding to study the impact of calcium. In addition, we characterized the pop2-5 mutant upon wounding and herbivore attack and, due to its inability to degrade GABA, employed this mutant line in transport studies with $\mathrm{D}_{2}$-labeled GABA.

\section{MATERIALS AND METHODS}

\section{Plant Growth and Treatment}

Four to 5-week-old plants grown in $10 \mathrm{~cm}$ round pots were used for all experiments. Arabidopsis thaliana Columbia-0 wild-type and mutant plants (tpc1-2 and pop2-5) were kept at short day conditions after stratification for 2 days at $4^{\circ} \mathrm{C}$. The growth chamber was adjusted to $50-60 \%$ humidity and $21^{\circ} \mathrm{C}$ with a 
10-h-light/14-h-dark photoperiod using FLUORA ${ }^{\circledR}$ L 36W/77 bulbs (OSRAM, Garching, Germany) with a light intensity of $100 \mu \mathrm{mol} \mathrm{m} \mathrm{m}^{-1}$. Seeds of $t p c 1-2$ were kindly provided by Prof. Edgar Peiter (University Halle) and pop2-5 seeds (GK_157D10) were purchased from GABI-Kat directly (Kleinboelting et al., 2012). To ensure the same starting conditions, all plants used for one assay were sown and germinated on the same day and were kept in the same growth chamber. For experiments investigating the systemic response and translocation of metabolites the leaves of each plant were counted according to their age (Dengler, 2006; Farmer et al., 2013; Kiep et al., 2015).

MecWorm treatment was used for mechanical wounding of the plant with punches every $5 \mathrm{~s}$, totaling 12 punches per minute on treated leaf 8 (Mithöfer et al., 2005; Scholz et al., 2015). To investigate the systemic response upon treatment of leaf 8 , the local and systemic leaves 5, 8, 9, 11, and/or 13 were analyzed. Untreated plants, kept exactly as the treated plants, were used as controls. The duration of the MecWorm treatment used is indicated in the respective figures. To study the woundinginduced systemic translocation of GABA in the plant, a doubledeuterated GABA (Sigma-Aldrich, Munich, Germany) was used and applied to pop2-5 mutant plants. The pop2-5 mutant was used for this assay since it cannot degrade GABA due to the knockout of the GABA-T gene (Palanivelu et al., 2003). According to (Jimenez-Aleman et al., 2015), $20 \mu \mathrm{l}$ of $50 \mu \mathrm{M} \mathrm{D}$-GABA or water was applied to local wounds on leaf 8 , which were generated with a pattern wheel (PRYM_610940, Prym, Stolberg, Germany) without wounding the midrib. On each side of the midrib, the leaf was wounded with six vertical motions. $\mathrm{D}_{2}$-GABA and watertreated plants were kept for 1.5 and $3 \mathrm{~h}$ with a cover to prevent evaporation. Samples of leaf 8 and chosen systemic leaves were harvested after the indicated time points. To avoid additional accumulation of GABA due to the cutting and harvesting process, all samples were harvested in less than 1 min and directly stored in liquid nitrogen. For all plants the leaves were harvested from oldest to youngest leaf (starting with leaf 5). All samples were kept at $-80^{\circ} \mathrm{C}$ till further analysis.

\section{Insect Material and Feeding Assays}

Larvae of the generalist herbivore Spodoptera littoralis were hatched and reared on artificial diet (Bergomaz and Boppre, 1986) at $23-25^{\circ} \mathrm{C}$ with 10 -h-light/14-h-dark cycles. For the 1 week long-term feeding assay, three larvae of first instar were placed on every wild-type and mutant plant. To achieve similar starting conditions, all larvae determined for one plant genotype were pooled and weighed prior to the experiment. The minimal starting weight of 30 larvae was set to $60 \mathrm{mg}$ to ensure a survival of all larvae. After 1 week the weight of every larva was recorded separately. For the short-term feeding assay third instar larvae were used after they were kept overnight without food. This treatment ensures an immediate start of feeding after placement on the plant. The locally fed leaves were collected after the indicated time points and kept at $-80^{\circ} \mathrm{C}$ till further analysis.

\section{Extraction and Quantification of GABA}

Two hundred and fifty milligram of fresh leaves were frozen in liquid nitrogen and weighed to enable a GABA determination per $g$ fresh weight. If multiple leaves of a plant were collected, then leaves were cut according to their age starting with the oldest (leaf 5). The leaf material was homogenized in a Geno/Grinder 2010 (Spex Sample Prep, Stanmore, UK) equipped with aluminum racks. The racks were cooled in liquid nitrogen prior to usage to prevent a thawing of leaf material during the whole homogenization process. The amino acids (including GABA) were extracted twice with a total of $2 \mathrm{ml}$ of methanol on ice. Supernatants were combined and dried using a Concentrator plus (Eppendorf, Hamburg, Germany) and re-suspended in $500 \mu \mathrm{l}$ of methanol. The extract was diluted 1:20 (v/v) with water containing the internal standard. The algal amino acid mix ${ }^{13} \mathrm{C},{ }^{15} \mathrm{~N}$ (Isotec, Miamisburg, OH, USA) was used as internal standard at a concentration of $10 \mu \mathrm{g}$ $\mathrm{ml}^{-1}$ in all samples. The concentration of unlabeled and $\mathrm{D}_{2}$ GABA $(\mathrm{M}+2)$ was analyzed by LC-MS/MS according to previous studies (Scholz et al., 2015). An API 5000 tandem mass spectrometer (Applied Biosystems, Darmstadt, Germany) was operated in positive ionization mode with multiple reaction monitoring (MRM) to monitor analyte parent ion $\rightarrow$ product ion: GABA $(m / z 104.1 \rightarrow 87.1$; DP 51 , CE 17); this MRM is specific for GABA and does not detect any $\alpha$-aminobutyric acid, $D_{2}$-GABA $(m / z 106.1 \rightarrow 89.1+m / z 106.1 \rightarrow 88.1$; DP 51, CE 17).

\section{Extraction and Quantification of Phytohormones}

Like the quantification of GABA, $250 \mathrm{mg}$ of leaf material was used for phytohormone analysis. The extraction procedure and determination of JA and JA-Ile was carried out on ice as described before (Vadassery et al., 2012) with small changes. In this study, a different mixture of labeled jasmonates was used as internal standard. Instead of $15 \mathrm{ng}$ of JA-[ $\left.{ }^{13} \mathrm{C} 6\right]-$ Ile conjugate used in the previous study, $60 \mathrm{ng}$ of $\mathrm{D}_{6}$-JA-Ile (HPC Standards $\mathrm{GmbH}$, Cunnersdorf, Germany) was used. Additionally, the $60 \mathrm{ng}$ of 9,10- $\mathrm{D}_{2}-9,10$-dihydrojasmonic acid was replaced by $60 \mathrm{ng}$ of $\mathrm{D}_{6}$ JA (HPC Standards GmbH, Cunnersdorf, Germany). Since it was observed that both the $\mathrm{D}_{6}$-labeled JA and JA-Ile contained $40 \%$ of the corresponding $\mathrm{D}_{5}$-labeled compounds (which were not included in the analysis method), the obtained results were divided by 1.7 to exclude this inaccuracy.

\section{Statistics}

All plants of different mutant lines used for one experiment were grown in the same growth chamber. Independent experiments were treated as a completely randomized design. Experiments were repeated three times to ensure reproducibility and 5-10 plants were used in each treatment for each experiment time point. Data of all independent experiments were pooled and analyzed. For comparison of two groups, the Student's $t$-test or Mann Whitney $U$-test was applied. For statistical analyses of multiple groups, one-way analysis of variance (one-way ANOVA) or two-way analysis of variance (two-way ANOVA) were used as indicated in the figure legends. Different letters indicate significant differences between treatments. GraphPad 


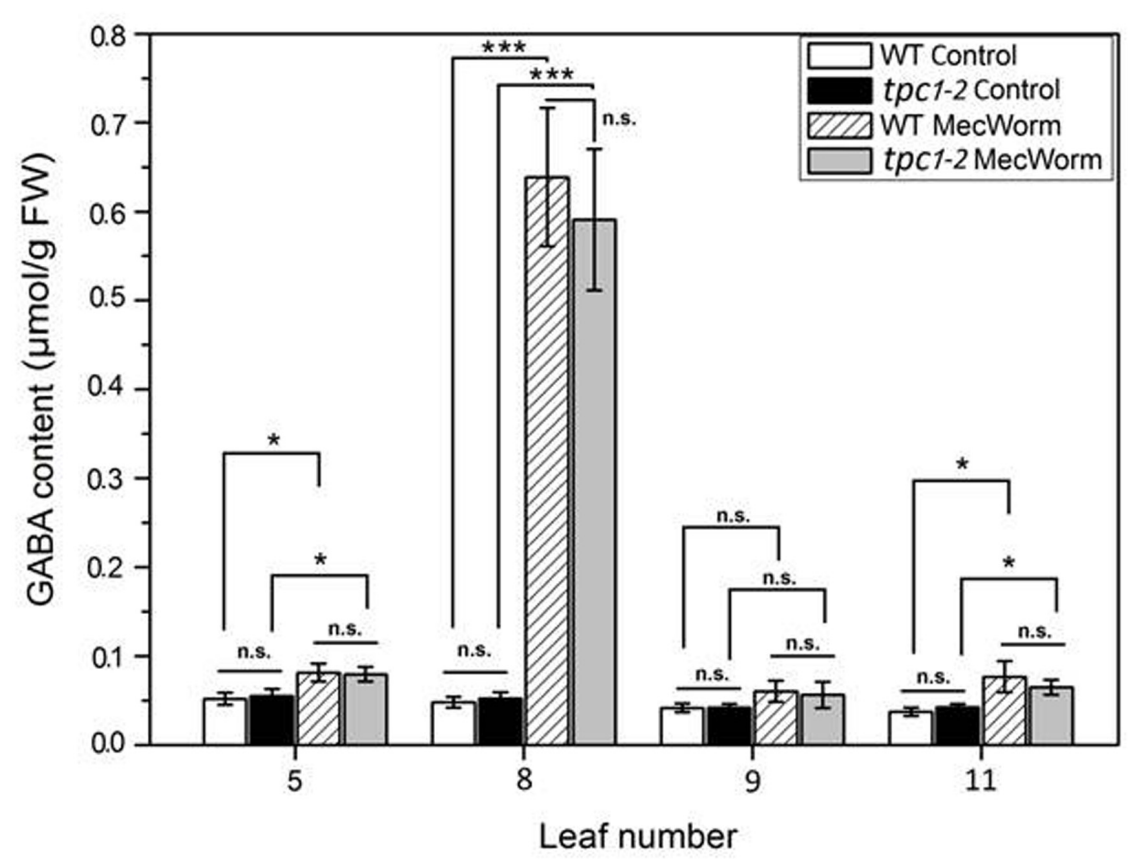

FIGURE 1 | Accumulation of GABA in individual Arabidopsis leaves of wild-type (WT) and tpc1-2 plants after MecWorm treatment. Mean [ \pm SE, $n=6$ (control), 8 (MecWorm)] levels of GABA were determined in individual leaves of untreated control plants and plants after $1.5 \mathrm{~h}$ treatment with MecWorm. In treated plants, leaf 8 was subjected to mechanical damage and systemic leaves 5, 9, and 11, and treated leaf 8 were analyzed for GABA level. Significant differences between the GABA level in the same leaf of the control and treated plant were analyzed by $t$-test (for each leaf separately, $p<0.05$, Mann-Whitney $U$-test), $* P \leq 0.05 ; * * * P \leq 0.001$.

Prism 6 and Origin Pro were used for data analysis and graph composition.

\section{RESULTS AND DISCUSSION}

\section{GABA Accumulates in Systemic Untreated Leaves in the Calcium-Channel Mutant tpc1-2}

To examine if the production of GABA in untreated systemic leaves is induced in a $\mathrm{Ca}^{2+} /$ calmodulin-dependent manner (Snedden et al., 1995), the systemic GABA accumulation was analyzed after MecWorm treatment in the tpc1-2 mutant. Recently, it was observed, that the tpc1-2 mutant did not show a systemic $\left[\mathrm{Ca}^{2+}\right]_{\text {cyt }}$ elevation after mechanical wounding (Kiep et al., 2015). Thus, tpc1-2 is a good model to study whether or not the systemic GABA accumulation depends on the systemic calcium signal. As expected, after $1.5 \mathrm{~h}$ of MecWorm treatment on WT leaf 8, this leaf showed the highest GABA accumulation. However, a significant increase in GABA concentration was also observed in systemic leaves 5 and 11 (Figure 1). With $0.05 \mu \mathrm{mol} \mathrm{g} \mathrm{FW}^{-1}$ the $t p c 1$ 2 plants showed the same basic GABA level in control plants as the WT and also a comparable increase in systemic leaves 5 and 11 (Figure 1). This indicates that the insectlike wounding-induced systemic $\left[\mathrm{Ca}^{2+}\right]_{\text {cyt }}$ elevation has no or limited influence on the amount of GABA produced in systemic leaves. For soybean GAD it was observed that an increase in $\left[\mathrm{Ca}^{2+}\right]_{\text {cyt }}$ up to $7-11 \mu \mathrm{M}$ is necessary to decrease the enzyme's Km value about 55\% (Snedden et al., 1995). The determined stress-induced $\left[\mathrm{Ca}^{2+}\right]_{\text {cyt }}$ elevations in Arabidopsis showed an increase to a maximum of 0.6-2 $\mu \mathrm{M}$ (Knight et al., 1996, 1997; Whalley and Knight, 2013). Hence, the woundinginduced systemic $\left[\mathrm{Ca}^{2+}\right]_{\text {cyt }}$ elevation appears to be insufficient to account for the increasing GAD activity observed in the present study.

\section{The GABA Accumulating pop2-5 Mutant Responds Efficiently to Mechanical Wounding and Spodoptera littoralis Feeding}

Due to the results obtained with tpc1-2 plants, the possibility of direct GABA transport to systemic leaves was investigated. Therefore, we employed the GABA-transaminase knock-out mutant line, pop2-5. This mutant has not been used and characterized in the context of herbivory. Previous studies observed that increased concentrations of GABA can affect insect's development (Ramputh and Bown, 1996; MacGregor et al., 2003; Bown et al., 2006; Scholz et al., 2015). We used the pop2-5 mutant to further evaluate the effect of high endogenous GABA concentration in planta on the herbivorous larvae of Spodoptera littoralis in a feeding assay (Figure 2). Our results demonstrate that the larvae, feeding 1 week on pop2-5 plants, gained significantly less weight than the larvae 


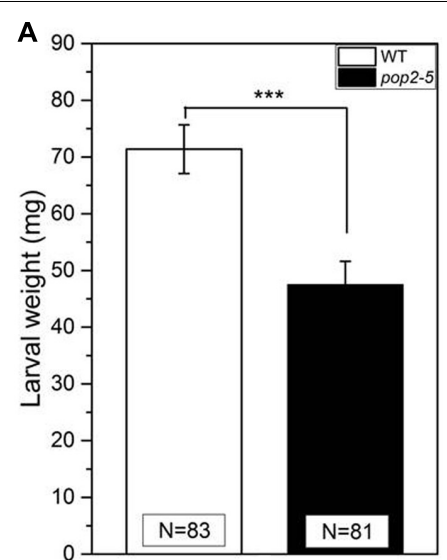

B

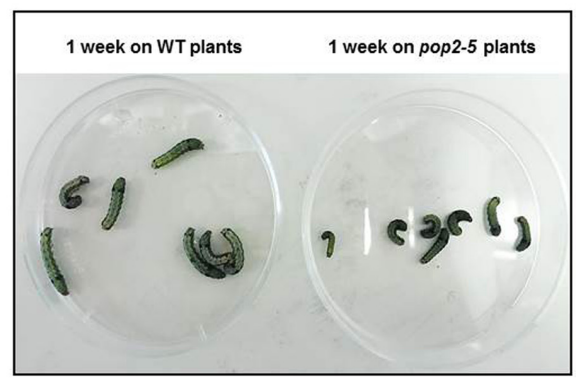

FIGURE 2 | Feeding assay of Spodoptera littoralis larvae on Arabidopsis WT and pop2-5 plants. S. littoralis first instar larvae were pre-weighed and three larvae were placed on each plant. The larval weight (mean $\pm \mathrm{SE}$ ) was measured after 7 days of feeding. (A) Final weight of larvae after feeding for 1 week on WT (white) and pop2-5 (black) plants. The total number of larvae weighed $(\mathrm{N})$ is indicated in the bars. (B) Larval macroscopic view after 1 week of feeding on the indicated mutants. Experiments were repeated three times independently. Significant differences between WT and GABA mutant plants after feeding were analyzed by Student's $t$-test $\left.{ }^{* * *} P \leq 0.001\right)$.

feeding on WT plants (Figure 2A). The difference could also be observed by macroscopic inspection (Figure 2B). In previous experiments with GABA-supplemented artificial diet it was shown, that larvae of $C$. rosaceana and $S$. littoralis feeding on high levels of GABA showed decreased survival, delayed development and decreased gain of weight, respectively (Ramputh and Bown, 1996; Scholz et al., 2015). This indicates that the constitutive GABA accumulation in pop2-5 plants seems to contribute to the enhanced resistance against $S$. littoralis larvae attack while the exact effect of GABA on the insects is not known. Enhanced GABA levels in the insect might lead to permanent activation of $\mathrm{GABA}$-activated $\mathrm{Cl}^{-}$-channels resulting in hypertension or paralysis (Sattelle, 1990; Bown et al., 2006).

We further investigated the GABA response upon MecWorm wounding in well-developed leaves of pop2-5 plants (Figure 3A). With MecWorm we are able to investigate the impact of the isolated wounding process without the contribution of insectderived compounds in oral secretions (Mithöfer et al., 2005). As shown in Figure 3B, continuous mechanical wounding of leaf 8 significantly elevated the amount of GABA in the treated leaf
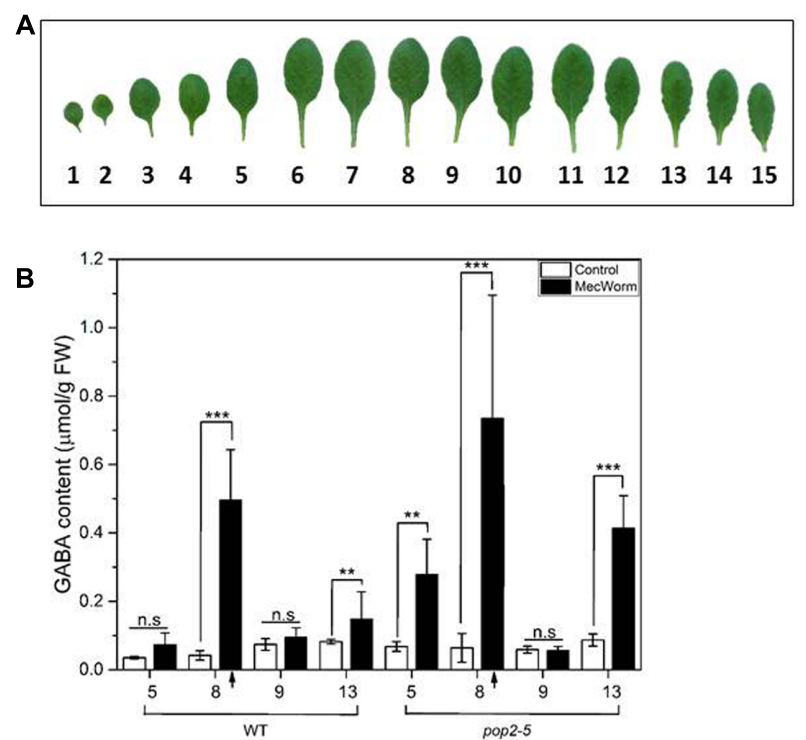

C

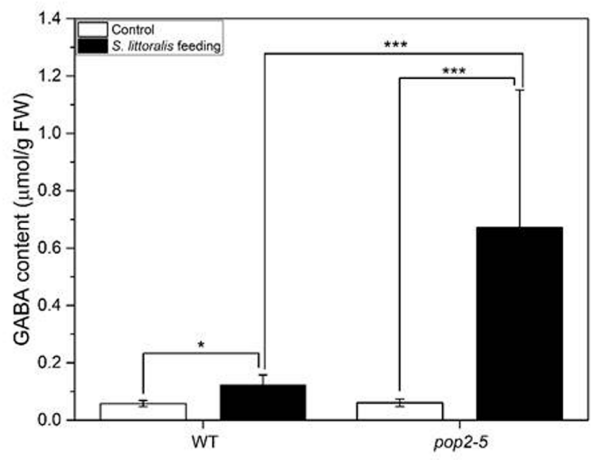

FIGURE 3 | Accumulation of GABA in individual Arabidopsis leaves after MecWorm treatment and Spodoptera littoralis short-term feeding assay. (A) Leaves of 4-week old Arabidopsis thaliana plants in growing order, from the oldest to the youngest. (B) Mean ( \pm SE, $n=5$ ) levels of GABA were determined in individual leaves of untreated control plants (white) and plants after treatment with MecWorm for $1.5 \mathrm{~h}$ (black). In treated plants, leaf 8 was subjected to mechanical damage (indicated by arrow) and systemic leaves 5, 9, and 11, and treated leaf 8 were analyzed for GABA level. (C) Mean $( \pm S E, n=5$ ) levels of GABA were determined in individual leaves of untreated control plants (white) and plants after feeding of third instar S. littoralis larvae for $1.5 \mathrm{~h}$ (black). Significant differences between the GABA level were analyzed by $t$-test (for each leaf separately, $p<0.05$,

Mann-Whitney $U$-test), ${ }^{*} P \leq 0.05$; ${ }^{*} P \leq 0.01 ;{ }^{* * *} P \leq 0.001$. No significant difference in GABA concentration was found between untreated genotypes.

in both WT and pop2-5 plants. For WT plants, apart from the local GABA accumulation, we observed a significant increase of GABA in leaf 13, which is directly connected to leaf 8 and a non-significant increase in the indirectly connected leaf 5 (Dengler, 2006). For pop2-5 plants the local GABA accumulation of leaf 8 was followed by a significant increase of GABA in leaves 5 and 13. In preliminary experiments we observed that the GABA elevation in the adjacent leaves is a time-dependent response, and after $90 \mathrm{~min}$ of feeding-like wounding of leaf 8 a significant increase of GABA was detectable in leaf 13. As expected, there was no significant increase of GABA on leaf 9 , 
A

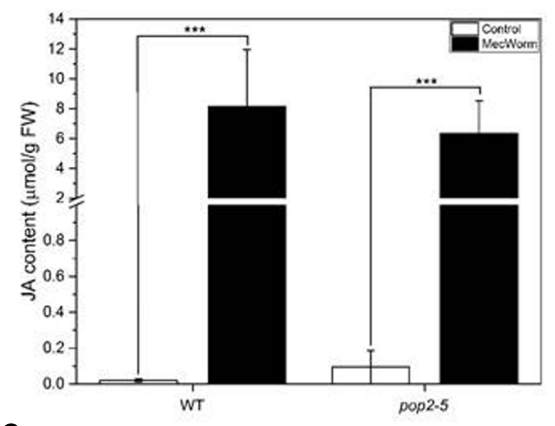

C

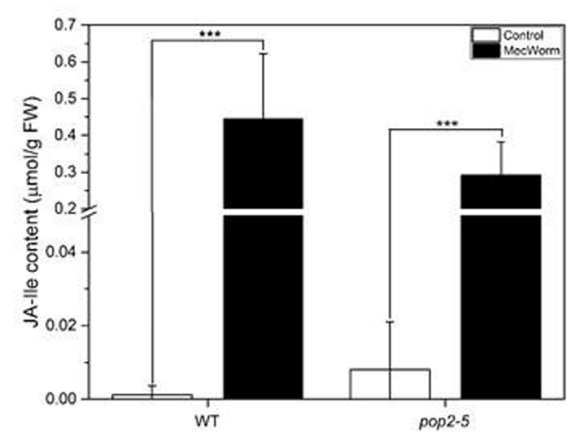

B

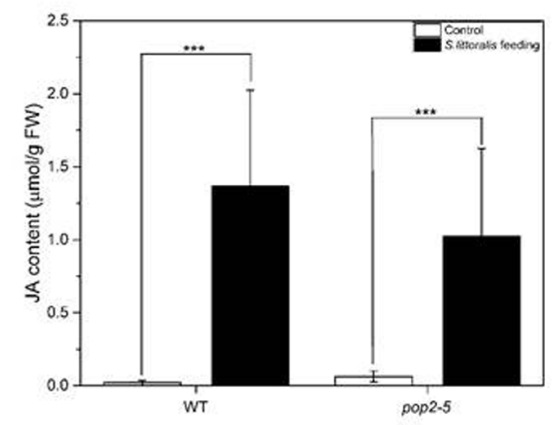

D

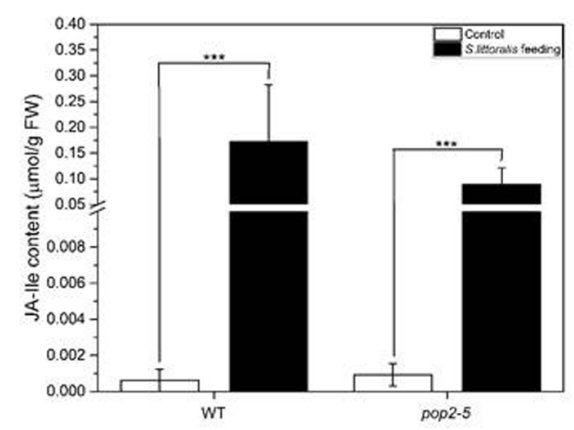

FIGURE 4 | Accumulation of jasmonates in Arabidopsis leaves after Spodoptera littoralis short time feeding assay and MecWorm treatment. JA levels were analyzed after mechanical wounding for $1.5 \mathrm{~h} \mathrm{(A)} \mathrm{and} \mathrm{after} \mathrm{S.} \mathrm{littoralis} \mathrm{feeding} \mathrm{for} 3 \mathrm{~h}$ (B). JA-lle levels were analyzed after mechanical wounding for $1.5 \mathrm{~h}$ (C) and after S. littoralis feeding for $3 \mathrm{~h}$ (D). For MecWorm treated plants, leaf 8 was subjected to mechanical damage and analyzed for jasmonate level. For quick feeding assay hormone level was analyzed from local fed leaves of third instar $S$. littoralis feeding for $1.5 \mathrm{~h}$. Untreated leaves from untreated plants were used as controls. Statistically significant differences between the JA and JA-lle levels of the control and treated plant were analyzed by Student's $t$-test (*** $P \leq 0.001$ ). No significant differences in JA and JA-lle levels were found between the analyzed genotypes.

as it is not connected to treated leaf 8 . Analysis of the GABA concentration after $1.5 \mathrm{~h}$ of $S$. littoralis feeding revealed that the larvae caused a 6-fold higher GABA accumulation in pop25 compared to WT plants, in the fed leaves (Figure $3 \mathrm{C}$ ). The synthesis of GABA in pop2-5 plants during treatments should be the same but in WT there is more GABA degradation by the transaminase during the assay, so the accumulation at the end was lower in the WT (Figure 3C). Here, it should be mentioned that in this set of experiments no clear difference in GABA concentrations between untreated WT and pop2-5 plants was found. This contradicts earlier findings including our own study (Palanivelu et al., 2003; Miyashita and Good, 2008; Clark et al., 2009; Scholz et al., 2015) where in pop2-5 plants a higher GABA level was detected. However, this might be explained by different growth conditions, i.e., long vs. short day. Nevertheless, taken together, the results indicate that also in pop2-5 mutant plants GABA is an efficient inducible systemic defense factor against insect feeding.

\section{Jasmonate Levels Are Not Affected in the pop2-5 Mutant}

The accumulation of jasmonates was not influenced by different GABA levels in $g a d 1 / 2$ and $\operatorname{gad} 1 / 2 \times$ pop2-5 mutants. Similarly the accumulation of GABA was not changed in jar1, a JA-Ile jasmonate signaling mutant (Scholz et al., 2015). We decided to further test the involvement of jasmonates on GABA accumulation also in the non-GABA-degrading pop2-5 mutant. Therefore we measured the levels of JA and its bioactive derivative, (+)-7-iso-jasmonoyl-L-isoleucine (JA-Ile), in Arabidopsis WT and in pop2-5 plants both upon mechanical wounding and herbivore treatment. The concentration of JA and JA-Ile significantly increased after feeding assay with $S$. littoralis and after MecWorm treatment in both tested genotypes (Figure 4). In all cases the MecWorm treatment caused higher jasmonate accumulation but the non-treated controls showed no differences between WT and pop2-5 plants. These data support earlier results suggesting that GABA accumulation is not jasmonate-dependent (Scholz et al., 2015) and vice versa. However, we cannot completely rule out that an octadecanoic dependent, but JA/JA-Ile independent, signaling pathway might still be involved in GABA accumulation.

\section{$D_{2}$-GABA Treatment Increases GABA Concentration in Systemic Adjacent Leaves}

To further investigate if GABA is transported to or synthetized in the adjacent leaves, we treated Arabidopsis pop2-5 plants 
A

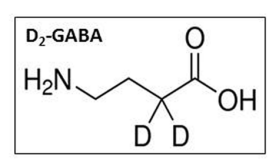

B

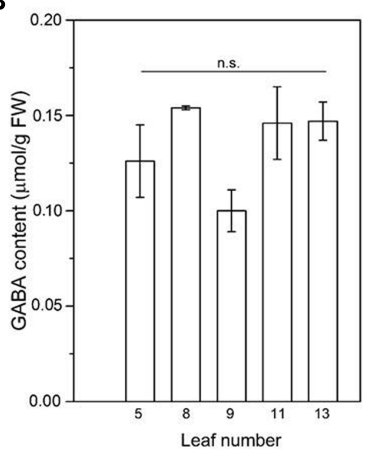

C

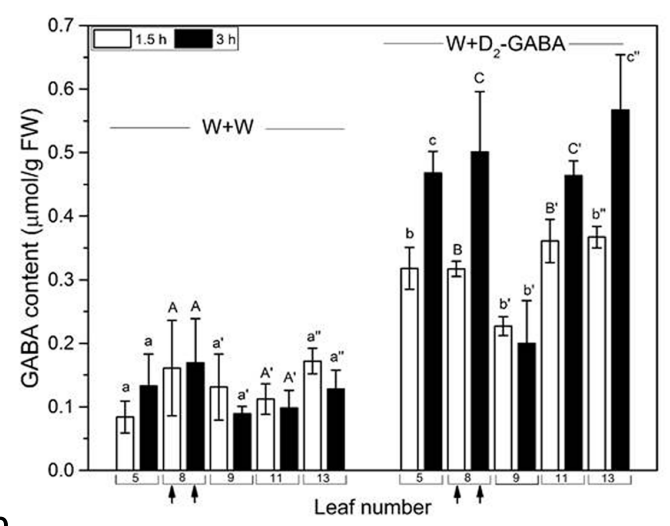

D

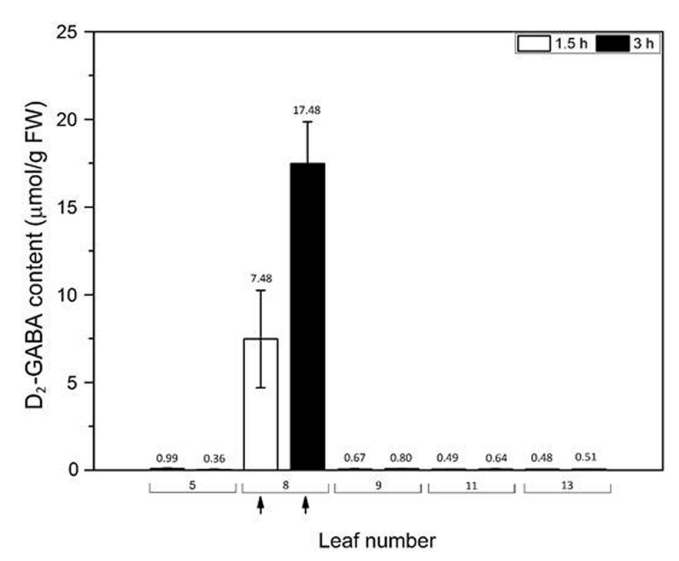

FIGURE 5 | Accumulation of GABA after isotopically labeled GABA ( $D_{2}$-GABA) treatment in pop2-5 plants. (A) Chemical structure of $\mathrm{D}_{2}$-GABA used. (B) Mean ( \pm SE, $n=7$ ) level of unlabeled GABA in untreated leaves from control plants. (C) Mean ( \pm SE, $n=7$ ) level of unlabeled GABA in pop2-5 plants was determined after wounding leaf 8 with a pattern wheel (indicated by arrow), applying water (wounding + water, $W+W$ ) or $D_{2}-G A B A$ (wounding $+D_{2}-G A B A, W+D_{2}-G A B A$ ) on the wounds and incubating for $1.5 \mathrm{~h}$ (white) and $3 \mathrm{~h}$ (black). No statistically significant difference was found between the controls (in B) and the wounding plus water treatment. (D) Mean ( \pm SE, $n=7$ ) levels of $D_{2}$-GABA were determined in different leaves after wounding leaf 8 with a pattern wheel (indicated by arrow), applying $D_{2}-G A B A$ on the wounds and incubating for $1.5 \mathrm{~h}$ (white) and $3 \mathrm{~h}$ (black). Values below $1 \mu \mathrm{mol}\left(\mathrm{g} \mathrm{FW}^{-1}\right.$ do not represent $\mathrm{D}_{2}$-GABA but an unknown endogenous compound and should be considered as noise. Significant differences between the treatments were analyzed by One-way ANOVA and Two-way ANOVA ( $p<0.05$, SNK-test) and are indicated by different letters.

with the double-deuterated $\mathrm{D}_{2}$-GABA (Figure 5A) on a leaf that was wounded with a pattern wheel. This approach would enable us to observe any translocation of GABA in the plant leaves, because no $\mathrm{D}_{2}$-GABA degradation takes place in the pop2-5 mutant. The basic GABA levels in different leaves of control pop2-5 plants were comparable (Figure 5B). Leaves of the pop2-5 plants that were slightly pattern wheel-wounded and incubated with water, showed a GABA concentration similar to control plants (Figures 5B,C), indicating that the weak single mechanical wounding had no effect compared to continuous mechanical wounding achieved with MecWorm treatment. Interestingly, when GABA was measured in leaf 8-wounded pop 2-5 plants that were treated with $\mathrm{D}_{2}-\mathrm{GABA}$, an increase of non-labeled GABA was detected, with the accumulation of GABA in directly connected (leaf 13) and indirectly connected (leaves 5 and 11) leaves. GABA accumulation was significantly higher after $1.5 \mathrm{~h}$ and increased more after $3 \mathrm{~h}$ (Figure 5C). Even leaf 9 showed, compared to wounding and water treatment $(\mathrm{W}+\mathrm{W})$, a slight elevation of unlabeled GABA after the application of $\mathrm{D}_{2}$-GABA ( $+\mathrm{D}_{2}$-GABA). These results suggest that GABA synthesis was stimulated by the combination of the pattern wheel wounding and $\mathrm{D}_{2}$ GABA incubation in both the treated leaf and systemic leaves (Figure 5C). When we further analyzed the concentration of $D_{2}$-GABA on pop2-5 treated plants it was only possible to detect higher $\mathrm{D}_{2}$-GABA level in the locally treated leaf 8 indicating that $\mathrm{D}_{2}$-GABA was taken up. However, the fact that in the adjacent leaves $\mathrm{D}_{2}$-GABA was detected not even nearly as in leaf 8 (Figure 5D) suggests that it is not transported from the local wounded leaf to the systemic leaves. This result, combined with the observation that also the systemic cytosolic calcium elevation after wounding is not responsible for activation of GABA synthesis in systemic unwounded leaves, demonstrates that there has to be a different signal initiating GABA synthesis and accumulation in systemic leaves. Previous studies in animals presented data concerning GABAmediated positive feedback loop (Kamermans and Werblin, 1992; Fenalti et al., 2007) and also in plants a several-fold endogenous accumulation of GABA after external application of GABA has been found for different tissues (Shi et al., 2010; Shang et al., 2011; Malekzadeh et al., 2014; Vijayakumari and Puthur, 2016). Further studies are required to establish a role for phloem B-type cells in long-distance signaling in plants (Shelp, 2012). In a study concerning drought stress tolerance with a Poaceae species, Agrostis stolonifera, GABA was shown to enhance the accumulation of GABA and of other amino acids (glycine, valine, proline, 5-oxoproline, serine, threonine, aspartic acid and glutamic acid) (Li et al., 2017). Interestingly, in barley plants it was shown that external application of GABA was able to induce action potentials (APs) accompanied by cytoplasmatic acidification short of the position of the stimulus (Felle and Zimmermann, 2007). APs and other electrophysiological reactions represent conceivable signaling candidates for systemic leaves because they have been shown to be involved in wound-induced leaf-to-leaf signaling (Zimmermann et al., 2009, 2016; Mousavi et al., 2013). 


\section{CONCLUSION}

Wounding of plant tissue and cell disruption caused by feeding insects demonstrated that GABA synthesis and accumulation can be a rapid defense response against invertebrate pests. Other studies proposed that GABA might have a role as signaling molecule, activated upon abiotic stresses. Our results demonstrate that under stress-conditions GABA cannot only act as a defense metabolite, but also as a signaling molecule. The approach with isotopically labeled GABA demonstrates in addition that a high local concentration of GABA in the challenged leaf can trigger a de novo synthesis of GABA in systemic untreated leaves. This systemic accumulation of GABA is neither dependent on the systemic cytosolic calcium elevation induced by the wounding nor on the direct transport of GABA from wounded to systemic leaves. The signal responsible for this observation remains still unclear but electrophysiological reactions might represent conceivable signaling candidates.

\section{REFERENCES}

Bergomaz, R., and Boppre, M. (1986). A simple instant diet for rearing arctiidae and other moths. J. Lepid. Soc. 40, 131-137.

Bouche, N., and Fromm, H. (2004). GABA in plants: just a metabolite? Trends Plant Sci. 9, 110-115. doi: 10.1016/j.tplants.2004.01.006

Bown, A. W., Hall, D. E., and MacGregor, K. B. (2002). Insect footsteps on leaves stimulate the accumulation of 4-aminobutyrate and can be visualized through increased chlorophyll fluorescence and superoxide production. Plant Physiol. 129, 1430-1434. doi: 10.1104/pp.006114

Bown, A. W., Macgregor, K. B., and Shelp, B. J. (2006). Gamma-aminobutyrate: defense against invertebrate pests? Trends Plant Sci. 11, 424-427. doi: 10.1016/ j.tplants.2006.07.002

Bown, A. W., and Shelp, B. J. (1997). The metabolism and functions of [gamma]-aminobutyric acid. Plant Physiol. 115, 1-5. doi: 10.1104/pp. 115.1.1

Bown, A. W., and Shelp, B. J. (2016). Plant GABA: not just a metabolite. Trends Plant Sci. 21, 811-813. doi: 10.1016/j.tplants.2016.08.001

Carroll, A. D., Fox, G. G., Laurie, S., Phillips, R., Ratcliffe, R. G., and Stewart, G. R. (1994). Ammonium assimilation and the role of $\gamma$-aminobutyric acid in $\mathrm{pH}$ homeostasis in carrot cell suspensions. Plant Physiol. 106, 513-520. doi: $10.2307 / 4276085$

Clark, S. M., Di Leo, R., Van Cauwenberghe, O. R., Mullen, R. T., and Shelp, B. J. (2009). Subcellular localization and expression of multiple tomato gammaaminobutyrate transaminases that utilize both pyruvate and glyoxylate. J. Exp. Bot. 60, 3255-3267. doi: 10.1093/jxb/erp161

Dengler, N. G. (2006). The shoot apical meristem and development of vascular architecture. Can. J. Bot. 84, 1660-1671. doi: 10.1139/b06-126

Fait, A., Fromm, H., Walter, D., Galili, G., and Fernie, A. R. (2008). Highway or byway: the metabolic role of the GABA shunt in plants. Trends Plant Sci. 13, 14-19. doi: 10.1016/j.tplants.2007.10.005

Farmer, E., Mousavi, S., and Lenglet, A. (2013). Leaf numbering for experiments on long distance signalling in Arabidopsis. Protoc. Exch. doi: 10.1038/protex. 2013.071

Felle, H. H., and Zimmermann, M. R. (2007). Systemic signalling in barley through action potentials. Planta 226, 203-214. doi: 10.1007/s00425-006-0458-y

Fenalti, G., Law, R. H., Buckle, A. M., Langendorf, C., Tuck, K., Rosado, C. J., et al. (2007). GABA production by glutamic acid decarboxylase is regulated by a dynamic catalytic loop. Nat. Struct. Mol. Biol. 14, 280-286. doi: 10.1038/ nsmb1228

Gilroy, S., Białasek, M., Suzuki, N., Górecka, M., Devireddy, A. R., Karpiński, S., et al. (2016). ROS. Calcium, and electric signals: key mediators of rapid systemic signaling in plants. Plant Physiol. 171, 1606-1615. doi: 10.1104/pp.16.00434

\section{AUTHOR CONTRIBUTIONS}

SS, JM, and AM conceived and designed the research; SS, JM, MR, and $\mathrm{MH}$ performed the experiments; SS, JM, FL, and AM wrote the manuscript. All authors contributed to the manuscript.

\section{ACKNOWLEDGMENTS}

We thank A. Lehr for assistance in the lab, A. Berg for rearing caterpillars and T. Krügel, A. Weber and the greenhouse team for growing plants. We thank Edgar Peiter (University Halle) for kindly providing $t p c 1-2$ seeds. We additionally thank Wilhelm Boland and the Max Planck Society and Celia R. Carlini for support. This work was further supported in part by the German Academic Exchange Service (DAAD; PPP Project ID 57142556) and by the Brazilian agency Coordenadoria de Aperfeiçoamento de Pessoal de Nível Superior (CAPES), Program PROBRAL 407/2016, grant 23038.006810/2014-51.

Jimenez-Aleman, G. H., Scholz, S. S., Heyer, M., Reichelt, M., Mithöfer, A., and Boland, W. (2015). Synthesis, metabolism and systemic transport of a fluorinated mimic of the endogenous jasmonate precursor OPC-8:0. Biochim. Biophys. Acta 12, 1545-1553. doi: 10.1016/j.bbalip.2015.09.002

Kamermans, M., and Werblin, F. (1992). GABA-mediated positive autofeedback loop controls horizontal cell kinetics in tiger salamander retina. J. Neurosci. 12, 2451-2463.

Kiep, V., Vadassery, J., Lattke, J., Maaß, J.-P., Boland, W., Peiter, E., et al. (2015). Systemic cytosolic Ca2+ elevation is activated upon wounding and herbivory in Arabidopsis. New Phytol. 207, 996-1004. doi: 10.1111/nph.13493

Kinnersley, A. M., and Turano, F. J. (2000). Gamma aminobutyric acid (GABA) and plant responses to stress. Crit. Rev. Plant Sci. 19, 479-509. doi: 10.1080/ 07352680091139277

Kleinboelting, N., Huep, G., Kloetgen, A., Viehoever, P., and Weisshaar, B. (2012). GABI-Kat SimpleSearch: new features of the Arabidopsis thaliana T-DNA mutant database. Nucleic Acids Res. 40, D1211-D1215. doi: 10.1093/nar/ gkr1047

Knight, H., Trewavas, A. J., and Knight, M. R. (1996). Cold calcium signaling in Arabidopsis involves two cellular pools and a change in calcium signature after acclimation. Plant Cell 8, 489-503. doi: 10.1105/tpc.8.3.489

Knight, H., Trewavas, A. J., and Knight, M. R. (1997). Calcium signalling in Arabidopsis thaliana responding to drought and salinity. Plant J. 12, 1067-1078. doi: 10.1046/j.1365-313X.1997.12051067.x

Li, Z., Yu, J., Peng, Y., and Huang, B. (2017). Metabolic pathways regulated by abscisic acid, salicylic acid and gamma-aminobutyric acid in association with improved drought tolerance in creeping bentgrass (Agrostis stolonifera). Physiol. Plant. 159, 42-58. doi: 10.1111/ppl.12483

MacGregor, K. B., Shelp, B. J., Peiris, S., and Bown, A. W. (2003). Overexpression of glutamate decarboxylase in transgenic tobacco plants deters feeding by phytophagous insect larvae. J. Chem. Ecol. 29, 2177-2182. doi: 10.1023/A: 1025650914947

Malekzadeh, P., Khara, J., and Heydari, R. (2014). Alleviating effects of exogenous Gamma-aminobutiric acid on tomato seedling under chilling stress. Physiol. Mol. Biol. Plants 20, 133-137. doi: 10.1007/s12298-013-0203-5

Mekonnen, D. W., Flügge, U. I., and Ludewig, F. (2016). Gamma-aminobutyric acid depletion affects stomata closure and drought tolerance of Arabidopsis thaliana. Plant Sci. 245, 25-34. doi: 10.1016/j.plantsci.2016.01.005

Mirabella, R., Rauwerda, H., Struys, E. A., Jakobs, C., Triantaphylides, C., Haring, M. A., et al. (2008). The Arabidopsis herl mutant implicates GABA in E-2hexenal responsiveness. Plant J. 53, 197-213. doi: 10.1111/j.1365-313X.2007. 03323.x

Mithöfer, A., Wanner, G., and Boland, W. (2005). Effects of feeding Spodoptera littoralis on lima bean leaves. II. Continuous mechanical wounding resembling 
insect feeding is sufficient to elicit herbivory-related volatile emission. Plant Physiol. 137, 1160-1168. doi: 10.1104/pp.104.054460

Miyashita, Y., and Good, A. G. (2008). Contribution of the GABA shunt to hypoxia-induced alanine accumulation in roots of Arabidopsis thaliana. Plant Cell Physiol. 49, 92-102. doi: 10.1093/pcp/pcm171

Mousavi, S. A. R., Chauvin, A., Pascaud, F., Kellenberger, S., and Farmer, E. E. (2013). GLUTAMATE RECEPTOR-LIKE genes mediate leaf-to-leaf wound signalling. Nature 500, 422-426. doi: 10.1038/nature12478

Nayyar, H., Kaur, R., Kaur, S., and Singh, R. (2014). $\gamma$-Aminobutyric acid (GABA) imparts partial protection from heat stress injury to rice seedlings by improving leaf turgor and upregulating osmoprotectants and antioxidants. J. Plant Growth Regul. 33, 408-419. doi: 10.1007/s00344-013-9389-6

Palanivelu, R., Brass, L., Edlund, A. F., and Preuss, D. (2003). Pollen tube growth and guidance is regulated by POP2, an Arabidopsis gene that controls GABA levels. Cell 114, 47-59. doi: 10.1016/S0092-8674(03)00479-3

Ramputh, A. I., and Bown, A. W. (1996). Rapid $\gamma$-aminobutyric acid synthesis and the inhibition of the growth and development of oblique-banded leaf-roller larvae. Plant Physiol. 111, 1349-1352. doi: 10.1104/pp.111.4.1349

Renault, H., El Amrani, A., Palanivelu, R., Updegraff, E. P., Yu, A., Renou, J. P., et al. (2011). GABA accumulation causes cell elongation defects and a decrease in expression of genes encoding secreted and cell wall-related proteins in Arabidopsis thaliana. Plant Cell Physiol. 52, 894-908. doi: 10.1093/pcp/pcr041

Sattelle, D. B. (1990). "GABA receptors of insects," in Advances in Insect Physiology, eds P. D. Evans and V. B. Wigglesworth (Cambridge, MA: Academic Press), 22.

Scholz, S. S., Reichelt, M., Mekonnen, D. W., Ludewig, F., and Mithöfer, A. (2015). Insect herbivory-elicited GABA accumulation in plants is a wound-induced. direct, systemic, and jasmonate-independent defense response. Front. Plant Sci. 6:1128. doi: 10.3389/fpls.2015.01128

Shang, H., Cao, S., Yang, Z., Cai, Y., and Zheng, Y. (2011). Effect of exogenous gamma-aminobutyric acid treatment on proline accumulation and chilling injury in peach fruit after long-term cold storage. J. Agric. Food Chem. 59, 1264-1268. doi: 10.1021/jf104424z

Shelp, B. J. (2012). Does long-distance GABA signaling via the phloem really occur? Botany 90, 897-900. doi: 10.1139/b2012-066

Shelp, B. J., Allan, W. L., and Faure, D. (2009). "Role of $\gamma$-aminobutyrate and $\gamma$-hydroxybutyrate in plant communication," in Plant-Environment Interactions, ed. F. Baluška (Berlin: Springer-Verlag), 73-84. doi: 10.1007/9783-540-89230-4_4

Shelp, B. J., Bozzo, G. G., Zarei, A., Simpson, J. P., Trobacher, C. P., and Allan, W. L. (2012). Strategies and tools for studying the metabolism and function of $\gamma$-aminobutyrate in plants. II. Integrated analysis. Botany 90, 781-793. doi: 10.1139/b2012-041
Shi, S. Q., Shi, Z., Jiang, Z. P., Qi, L. W., Sun, X. M., Li, C. X., et al. (2010). Effects of exogenous GABA on gene expression of Caragana intermedia roots under $\mathrm{NaCl}$ stress: regulatory roles for $\mathrm{H}_{2} \mathrm{O}_{2}$ and ethylene production. Plant Cell Environ. 33, 149-162. doi: 10.1111/j.1365-3040.2009.02065.x

Snedden, W. A., Arazi, T., Fromm, H., and Shelp, B. J. (1995). Calcium/calmodulin activation of soybean glutamate decarboxylase. Plant Physiol. 108, 543-549. doi: 10.1111/j.1365-3040.2009.02065.x

Vadassery, J., Reichelt, M., Hause, B., Gershenzon, J., Boland, W., and Mithöfer, A. (2012). CML42-mediated calcium signaling coordinates responses to Spodoptera herbivory and abiotic stresses in Arabidopsis. Plant Physiol. 159, 1159-1175. doi: 10.1104/pp.112.198150

Vijayakumari, K., and Puthur, J. T. (2016). $\gamma$-Aminobutyric acid (GABA) priming enhances the osmotic stress tolerance in Piper nigrum Linn. plants subjected to PEG-induced stress. Plant Growth Regul. 78, 57-67. doi: 10.1007/s10725-0150074-6

Wallace, W., Secor, J., and Schrader, L. E. (1984). Rapid accumulation of $\gamma$-aminobutyric acid and alanine in soybean leaves in response to an abrupt transfer to lower temperature, darkness, or mechanical manipulation. Plant Physiol. 75, 170-175. doi: 10.1007/s10725-015-0074-6

Whalley, H. J., and Knight, M. R. (2013). Calcium signatures are decoded by plants to give specific gene responses. New Phytol. 197, 690-693. doi: 10.1111/nph. 12087

Zimmermann, M. R., Maischak, H., Mithöfer, A., Boland, W., and Felle, H. H. (2009). System potentials, a novel electrical long-distance apoplastic signal in plants. induced by wounding. Plant Physiol. 149, 1593-1600. doi: 10.1104/pp. 108.133884

Zimmermann, M. R., Mithöfer, A., Will, T., Felle, H. H., and Furch, A. C. (2016). Herbivore-triggered electrophysiological reactions: candidates for systemic signals in higher plants and the challenge of their identification. Plant Physiol. 170, 2407-2419. doi: 10.1104/pp.15.01736

Conflict of Interest Statement: The authors declare that the research was conducted in the absence of any commercial or financial relationships that could be construed as a potential conflict of interest.

Copyright (C) 2017 Scholz, Malabarba, Reichelt, Heyer, Ludewig and Mithöfer. This is an open-access article distributed under the terms of the Creative Commons Attribution License (CC BY). The use, distribution or reproduction in other forums is permitted, provided the original author(s) or licensor are credited and that the original publication in this journal is cited, in accordance with accepted academic practice. No use, distribution or reproduction is permitted which does not comply with these terms. 